\title{
Quasiminimizing properties of solutions to Riccati type equations
}

\author{
OLli MARTIO
}

\begin{abstract}
Solutions $u$ of the Riccati equation $-\nabla \cdot A(x, \nabla u)=b(x)|\nabla u|^{q}$ with $A(x, h) \cdot h \approx|h|^{p}$ and $b$ a bounded function are studied in an open set $\Omega \subset \mathbf{R}^{n}$. It is shown that the solutions $u$ are local quasiminimizers whenever $p-1 \leq q \leq p$ for $p>n$ and $n-1 \leq q<n$ for $p=n$. This extends the results in the author's earlier paper [8] where the case $p<n$ was studied. Continuous solutions in the range $p / n+p-1 \leq q \leq p$ are also local quasiminimizers. Examples show that the results are quite sharp.
\end{abstract}

Mathematics Subject Classification (2010): $35 \mathrm{~J} 60$ (primary); $35 \mathrm{~J} 25$ (secondary).

\section{Introduction}

We consider solutions $u$ of the equation

$$
-\nabla \cdot A(x, \nabla u)=b(x)|\nabla u|^{q}
$$

where $A(x, h) \cdot h \approx|h|^{p}, p>1$. For the precise assumptions on $p, A, b$ and $q$ see (1.3)-(1.7) below. The solutions $u$ are understood in the weak sense. Hence $u$ is a solution of (1.1) in an open set $\Omega \subset \mathbf{R}^{n}$ if $u$ belongs to the local Sobolev space $W_{\text {loc }}^{1, p}(\Omega)$ and

$$
\int_{\Omega} A(x, \nabla u) \cdot \nabla \varphi d x=\int_{\Omega} \varphi b(x)|\nabla u|^{q} d x
$$

for all $\varphi \in C_{0}^{\infty}(\Omega)$. In Section 4 we consider solutions in the case $q>p$. Then we assume that a solution $u$ belongs to $W_{\mathrm{loc}}^{1, q}(\Omega)$.

We use the following assumptions in the sequel unless otherwise stated:

$$
b \text { is a bounded measurable function in } \Omega
$$


and $A: \Omega \times \mathbf{R}^{n} \rightarrow \mathbf{R}^{n}$ is a Caratheodory function such that for all $h \in \mathbf{R}^{n}$ and a.e. $x \in \Omega$

$$
\begin{aligned}
& A(x, h) \cdot h \geq \alpha|h|^{p}, \\
& |A(x, h)| \leq \beta|h|^{p-1}
\end{aligned}
$$

where $0<\alpha \leq \beta<\infty$. In Section 2 for the exponents $p>1$ and $q$ we use two sets of assumptions:

$$
p>n, p-1 \leq q \leq p
$$

and

$$
p=n, n-1 \leq q<n .
$$

In [8] the range

$$
q<p<n, p-1 \leq q \leq p / n+p-1,
$$

was studied and the purpose of this paper is to complete the picture of quasiminimizing properties of solutions to include the cases (1.6) and (1.7).

The prototype of (1.1) is the equation

$$
-\Delta_{p} u=-\nabla \cdot\left(|\nabla u|^{p-2} \nabla u\right)=|\nabla u|^{q}
$$

and the examples in Section 4 concern this much studied equation, see [9] for $q>p$ and for other values [1] and references therein.

We recall the concept of a quasiminimizer. Let $\Omega$ be an open subset of $\mathbf{R}^{\mathbf{n}}$, $n \geq 1, p>1$ and $K \geq 1$. A function $u$ in the local Sobolev space $W_{\text {loc }}^{1, p}(\Omega)$ is called a $(p, K)$-quasiminimizer in $\Omega$ if for all open sets $\Omega^{\prime} \subset \subset \Omega$

$$
\int_{\Omega^{\prime}}|\nabla u|^{p} d x \leq K \int_{\Omega^{\prime}}|\nabla v|^{p} d x
$$

for all functions $v$ such that $v-u \in W_{0}^{1, p}\left(\Omega^{\prime}\right)$. Note that if a function $u$ belongs to $W^{1, p}(\Omega)$, then $u$ is a $K$-quasiminimizer if and only if (1.10) holds for all open sets $\Omega^{\prime} \subset \Omega$, i.e. $\bar{\Omega}^{\prime}$ need not be a compact subset of $\Omega$. In general we keep the number $p$ fixed and use the abbreviation a $K$-quasiminimizer. For $K=1$ the function $u$ is minimizer and hence a $p$-harmonic function, i.e. $u$ satisfies $\Delta_{p} u=0$. For the properties of quasiminimizers see $[2,3,8]$ and references therein. Roughly speaking, the quasiminimizing property of $u$ means that $u$ is close to a solution of the $p$-harmonic equation. For the Riccati equation this means that the source term $b(x)|\nabla u|^{q}$ does not contribute much to the behavior of $u$.

We say that a function $u$ in $\Omega$ is a local $K$-quasiminimizer if every $x \in \Omega$ has a neighborhood $U$ such that $u \mid U$ is a $K$-quasiminimizer. The function $u$ is called a $K$ quasiminimizer in small sets in $\Omega$ if there is $\delta>0$ such that $u$ is $K$-quasiminimizer in every open set $\Omega^{\prime} \subset \Omega$ whenever $m\left(\Omega^{\prime}\right)<\delta$.

Our result in the next section says that all solutions $u \in W^{1, p}(\Omega)$ to (1.1) under the assumptions (1.3)-(1.5) and either (1.6) or (1.7) are quasiminimizers in small 
sets in $\Omega$. We then use this property and its local counterpart to derive uniqueness results for solutions. In Section 3 we show that continuous solutions in the range $p / n+p-1<q \leq p$ are local quasiminimizers as well. The method here is similar to that in [8]. The exponents $p$ and $q$ such that the equation (1.9) has solutions which are not local quasiminimizers are considered in Section 4.

\section{Main results}

We first consider the case where a solution $u$ of (1.1) belongs to $W^{1, p}(\Omega)$. Let

$$
D_{p}(u)=D_{p}(u, \Omega)=\int_{\Omega}|\nabla u|^{p} d x
$$

denote the $p$-Dirichlet integral of $u$ in $\Omega$.

Theorem 2.1. Suppose that $u \in W^{1, p}(\Omega)$ is a solution of the equation (1.1) in an open set $\Omega \subset \mathbf{R}^{n}$ where $A$ and $b$ satisfy the assumptions (1.3)-(1.5) and $p$ and $q$ the assumption (1.6), i.e. $p>n, p-1 \leq q \leq p$. Then $u$ is a quasiminimizer in small sets in $\Omega$. More precisely, there is $\delta=\delta\left(p, q, n, \alpha, M, D_{p}(u)\right)>0$ such that $u$ is a $(2 \beta / \alpha)^{p}$-quasiminimizer in $\Omega^{\prime} \subset \Omega$ whenever $m\left(\Omega^{\prime}\right)<\delta$. Here $M=\operatorname{ess} \sup _{\Omega}|b|$. In the case $q=p-1$ the number $\delta$ does not depend on $D_{p}(u)$.

Proof. Let $\Omega^{\prime} \subset \Omega$ be an open set and let $P=P\left(u, \Omega^{\prime}\right)$ be the function which minimizes the $p$-Dirichlet integral with boundary values $u$ in $\Omega^{\prime}$, i.e.

$$
\int_{\Omega^{\prime}}|\nabla P|^{p} d x=\inf _{v} \int_{\Omega^{\prime}}|\nabla v|^{p} d x
$$

over all functions $v-u \in W_{0}^{1, p}\left(\Omega^{\prime}\right)$. Such a unique $p$-harmonic function always exists, see e.g. [5, Chapter 5]. We use $u-P$ as a test function for the equation (1.2). This gives

$$
\int_{\Omega^{\prime}} A(x, \nabla u) \cdot \nabla(u-P) d x=\int_{\Omega^{\prime}}(u-P) b(x)|\nabla u|^{q} d x .
$$

We estimate the left and the right hand side of (2.1) separately.

For the left hand side we first use (1.4), (1.5) and the Hölder inequality to obtain

$$
\begin{aligned}
& \int_{\Omega^{\prime}} A(x, \nabla u) \cdot \nabla(u-P) d x \\
& \quad \geq \alpha \int_{\Omega^{\prime}}|\nabla u|^{p} d x-\beta\left(\int_{\Omega^{\prime}}|\nabla u|^{p} d x\right)^{(p-1) / p}\left(\int_{\Omega^{\prime}}|\nabla P|^{p} d x\right)^{1 / p} \\
& =\left(\int_{\Omega^{\prime}}|\nabla u|^{p} d x\right)^{(p-1) / p}\left(\alpha\left(\int_{\Omega^{\prime}}|\nabla u|^{p} d x\right)^{1 / p}-\beta\left(\int_{\Omega^{\prime}}|\nabla P|^{p} d x\right)^{1 / p}\right) .
\end{aligned}
$$


To estimate the right hand side of (2.1) we use

$$
\operatorname{ess} \sup _{\Omega}|w| \leq c m\left(\Omega^{\prime}\right)^{(p-n) /(n p)}\|\nabla w\|_{p}
$$

valid for a function $w \in W_{0}^{1, p}\left(\Omega^{\prime}\right)$ in the case $p>n$, see [4, Theorem 7.10]. The generic constant $c$ depends only on $p$ and $n$. Since

$$
\int_{\Omega^{\prime}}|\nabla u|^{p} d x \geq \int_{\Omega^{\prime}}|\nabla P|^{p} d x
$$

by the minimizing property of $P$, we get, after an application of the Hölder inequality, that

$$
\begin{aligned}
& \int_{\Omega^{\prime}}(u-P) b(x)|\nabla u|^{q} d x \\
& \leq c M m\left(\Omega^{\prime}\right)^{(p-n) / n p}\left(\int_{\Omega^{\prime}}|\nabla u|^{p} d x\right)^{1 / p}\left(\int_{\Omega^{\prime}}|\nabla u|^{p} d x\right)^{q / p} m\left(\Omega^{\prime}\right)^{(p-q) / p} \\
& =c M m\left(\Omega^{\prime}\right)^{(p-n+n(p-q)) / n p}\left(\int_{\Omega^{\prime}}|\nabla u|^{p} d x\right)^{(1+q) / p} .
\end{aligned}
$$

Note that we have used the assumption $q \leq p$ here.

Combining (2.2) and (2.4) we obtain

$$
\begin{aligned}
& \alpha\left(\int_{\Omega^{\prime}}|\nabla u|^{p} d x\right)^{1 / p}-\beta\left(\int_{\Omega^{\prime}}|\nabla P|^{p} d x\right)^{1 / p} \\
& \quad \leq c M m\left(\Omega^{\prime}\right)^{(p-n+n(p-q)) / n p}\left(\int_{\Omega^{\prime}}|\nabla u|^{p} d x\right)^{(2+q-p) / p} .
\end{aligned}
$$

Since $1+q-p \geq 0$, we get

$$
\left(\int_{\Omega^{\prime}}|\nabla u|^{p} d x\right)^{(2+q-p) / p} \leq D_{p}(u)^{(1+q-p) / p}\left(\int_{\Omega^{\prime}}|\nabla u|^{p} d x\right)^{1 / p}
$$

and choosing $\Omega^{\prime}$ so small that

$$
c M D_{p}(u)^{(1+q-p) / p} m\left(\Omega^{\prime}\right)^{(p-n+n(p-q)) / n p} \leq \alpha / 2
$$

we obtain from (2.5) and (2.6) that

$$
\int_{\Omega^{\prime}}|\nabla u|^{p} d x \leq(2 \beta / \alpha)^{p} \int_{\Omega^{\prime}}|\nabla P|^{p} d x .
$$

This means that $u$ satisfies (1.10) with $K=(2 \beta / \alpha)^{p}$. Hence $u$ is a $K$-quasiminimizer in small sets in $\Omega$. More precisely, there is $\delta=\delta\left(p, q, n, \alpha, M, D_{p}(u)\right)>$ 0 such that $u$ is a $K$-quasiminimizer in $\Omega^{\prime} \subset \Omega$ whenever $m\left(\Omega^{\prime}\right)<\delta$. In the case $q=p-1$ the number $\delta$ does not depend on $D_{p}(u)$. The proof is complete. 
Remark 2.2. A look at the proof of the previous theorem shows that for the $p$ harmonic operator $A(x, h)=|h|^{p-2} h$, where $\alpha=\beta=1$, the number $K$ can be chosen arbitrary close to 1 by choosing $\delta$ small.

The proof of the above theorem immediately produces the following local version.

Theorem 2.3. Suppose that $u \in W_{\mathrm{loc}}^{1, p}(\Omega)$ is a solution of the equation (1.1) in an open set $\Omega \subset \mathbf{R}^{n}$ where $p, q$ and $A$ satisfy the assumptions (1.4)-(1.6) and $b$ is a locally bounded measurable function in $\Omega$. Then $u$ is a local quasiminimizer in $\Omega$. More precisely, for each $x \in \Omega$ there is $r>0$, such that $u$ is a $(2 \beta / \alpha)^{p}$-quasiminimizer in $B(x, r)$. The number $r$ depends only on $p, q, n, \alpha$,

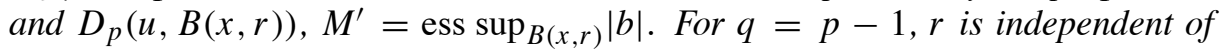
$D_{p}(u, B(x, r))$.

Next we consider the case $p=n$. The method is much the same as in Theorem 2.1 except a few twists.

Theorem 2.4. Suppose that $u \in W^{1, n}(\Omega)$ is a solution of the equation (1.1) in an open set $\Omega \subset \mathbf{R}^{n}$ where Let $A$ and $b$ satisfy the assumptions (1.3)-(1.5) and $p$ and $q$ the assumption (1.7), i.e. $p=n, n-1 \leq q<n$. Then $u$ is a quasiminimizer in small sets in $\Omega$. More precisely, there is $\delta=\delta\left(p, q, n, \alpha, M, D_{n}(u)>0\right.$ such that $u$ is a $(2 \beta / \alpha)^{n}$-quasiminimizer in $\Omega^{\prime} \subset \Omega$ whenever $m\left(\Omega^{\prime}\right)<\delta$. Here $M=$ ess $\sup _{\Omega}|b|$. In the case $q=n-1$ the number $\delta$ is independent of $D_{n}(u)$.

Proof. Fix an open set $\Omega^{\prime} \subset \Omega$ and let $P$ be the unique $n$-harmonic function in $\Omega^{\prime}$ with $u-P \in W_{0}^{1, p}\left(\Omega^{\prime}\right)$. As in the proof of Theorem 2.1 we use the function $u-P$ as a test function in the equation (1.2). Since $q<n$ the Hölder inequality gives for the right hand side of (1.2) an estimate

$$
\begin{aligned}
& \int_{\Omega^{\prime}}(u-P) b(x)|\nabla u|^{q} d x \\
& \quad \leq M\left(\int_{\Omega^{\prime}}|u-P|^{n /(n-q)} d x\right)^{(n-q) / n}\left(\int_{\Omega^{\prime}}|\nabla u|^{n} d x\right)^{q / n} .
\end{aligned}
$$

Next we write the exponent $n /(n-q)$ as $n s /(n-s)$ where $s=n /(n-q+1)$. Note that $s<n$ because $n>q$. Now we can use the Sobolev imbedding theorem, see e.g. [4, Theorem 7.10]. This yields

$$
\int_{\Omega^{\prime}}|u-P|^{n s /(n-s)} d x \leq c\left(\int_{\Omega^{\prime}}|\nabla(u-P)|^{s} d x\right)^{n /(n-s)}
$$

where $c$ is a generic constant depending only on $q$ and $n$ and we obtain from (2.7)

$$
\int_{\Omega^{\prime}}(u-P) b(x)|\nabla u|^{q} d x \leq c M\left(\int_{\Omega^{\prime}}|\nabla u|^{s} d x\right)^{(n-q) /(n-s)}\left(\int_{\Omega^{\prime}}|\nabla u|^{n} d x\right)^{q / n} .
$$


Using the Hölder inequality, then the the minimizing property (2.3) of $P$ for $p=n$ and taking the value of $s$ into account we get

$$
\begin{aligned}
\int_{\Omega^{\prime}} & (u-P) b(x)|\nabla u|^{q} d x \\
& \leq c M m\left(\Omega^{\prime}\right)^{\delta^{\prime}}\left(\int_{\Omega^{\prime}}|\nabla u|^{n} d x\right)^{s(n-q) / n(n-s)}\left(\int_{\Omega^{\prime}}|\nabla u|^{n} d x\right)^{q / n} \\
& =c M m\left(\Omega^{\prime}\right)^{\delta^{\prime}}\left(\int_{\Omega^{\prime}}|\nabla u|^{n} d x\right)^{(1+q) / n}
\end{aligned}
$$

where $\delta^{\prime}=(n-q) /(n-q+1)>0$.

We complete the proof as follows. Since $\nabla u \in L^{n}(\Omega)$ and $\delta^{\prime}>0$ there is $\delta=\delta\left(n, q, \alpha, M, D_{n}(u)\right)>0$ such that

$$
c M m\left(\Omega^{\prime}\right)^{\delta^{\prime}} D_{n}(u)^{(q-(n-1)) / n} \leq \alpha / 2
$$

whenever $m\left(\Omega^{\prime}\right)<\delta$ and hence

$$
\begin{array}{rl}
c M & m\left(\Omega^{\prime}\right)^{\delta^{\prime}}\left(\int_{\Omega^{\prime}}|\nabla u|^{n} d x\right)^{(1+q) / n} \\
& \leq c M m\left(\Omega^{\prime}\right)^{\delta^{\prime}}\left(D_{n}(u)\right)^{(q-(n-1)) / n} \int_{\Omega^{\prime}}|\nabla u|^{n} d x \leq \alpha / 2 \int_{\Omega^{\prime}}|\nabla u|^{n} d x .
\end{array}
$$

Thus from (2.1) and (2.2) for $p=n$ and from (2.9) we obtain

$$
\int_{\Omega^{\prime}}|\nabla u|^{n} d x \leq(2 \beta / \alpha)^{p} \int_{\Omega^{\prime}}|\nabla P|^{n} d x .
$$

This is (1.10) for $p=n$ and $K=(2 \beta / \alpha)^{p}$. Hence $u$ is a $K$-quasiminimizer in small sets in $\Omega$ as required. In the case $q=n-1$ the exponent $(q-(n-1)) / n=0$ and $\delta$ is independent of $D_{n}(u)$. The proof follows.

There is a local version of Theorem 2.4 corresponding to Theorem 2.3. It is similar to Theorem 2.3 and the formulation is left to the reader.

Since quasiminimizers satisfy the minimum and maximum principles, see $e . g$. [2, Theorem 2.3] and its counterpart for $p=n$ give this principle for the solutions of (1.1) which are local quasiminimizers. By the maximum principle we mean the strong maximum principle: If a function $u$ attains its maximum at the point $x_{o} \in \Omega$, then $u(x)=u\left(x_{o}\right)$ for all $x$ in the $x_{o}$-component of $\Omega$.

Corollary 2.5. Let $u$ be as in Theorem 2.3 or in the corresponding theorem for $p=n$. Then $u$ satisfies the maximum and minimum principles in $\Omega$.

If $u \in W_{0}^{1, p}(\Omega)$ is a quasiminimizer in small sets in $\Omega$, then $u=0$ in $\Omega$, see $[8$, Lemma 3.8]. Hence we obtain

Corollary 2.6. Let $u \in W_{0}^{1, p}(\Omega)$ be a solution of the equation (1.1) where $p, q$, $A$ and $b$ satisfy the assumptions (1.3)-(1.5) and either (1.6) or (1.7) in an open set $\Omega \subset \mathbf{R}^{n}$. Then $u=0$. 


\section{Continuous solutions}

Solutions of (1.1) need not be continuous and then they are not local quasiminimizers since local quasiminimizers are locally Hölder continuous. However, we show that in the range

$$
p / n+p-1 \leq q \leq p
$$

all continuous solutions are local quasiminimizers. Note that for $p-1 \leq q \leq$ $p / n+p-1, p<n$, all solutions are local quasiminimizers, see [8].

Theorem 3.1. Suppose that $u$ is a continuous solution of the equation (1.1) in an open set $\Omega \subset \mathbf{R}^{n}$ where $b \in L_{\mathrm{loc}}^{\infty}(\Omega)$ and A satisfies the assumptions (1.4)-(1.5) and $p$ and $q$ the assumption (3.1). Then $u$ is a local quasiminimizer in $\Omega$. More precisely, for each point $x_{o} \in \Omega$ there is $r>0$ depending on the modulus of continuity of $u$ in $B\left(x_{o}, r\right), D_{p}\left(u, B\left(x_{o}, r\right)\right)$, ess $\sup _{B(x, r)}|b|, n, p$ and $q$ such that $u \mid B\left(x_{o}, r\right)$ is a $(2 \beta / \alpha)^{p}$-quasiminimizer in $B\left(x_{o}, r\right)$. For $p=q$ the radius $r$ does not depend on $D_{p}\left(u, B\left(x_{o}, r\right)\right)$.

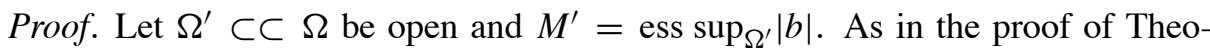
rem 2.1 we obtain an estimate

$$
\begin{aligned}
& \alpha \int_{\Omega^{\prime}}|\nabla u|^{p} d x-\beta\left(\int_{\Omega^{\prime}}|\nabla u|^{p} d x\right)^{(p-1) / p}\left(\int_{\Omega^{\prime}}|\nabla P|^{p} d x\right)^{1 / p} \\
& \quad \leq \int_{\Omega^{\prime}} A(x, \nabla u) \cdot \nabla(u-P) d x=\int_{\Omega^{\prime}}(u-P)|\nabla u|^{q} d x \\
& \quad \leq M^{\prime} \int_{\Omega^{\prime}}|u-P||\nabla u|^{q} d x .
\end{aligned}
$$

Let first $q<p$. Then by (3.1), $p<n$ and $\gamma=n(p-q) /(n-p) \in(0,1)$ and since $\gamma p /(p-q)=n p /(n-p)$, we obtain from the the Sobolev imbedding theorem, see $e . g$. [4, Theorem 7.10], and the Hölder inequality

$$
\begin{aligned}
& M^{\prime} \int_{\Omega^{\prime}}|u-P||\nabla u|^{q} d x \leq M^{\prime} \sup _{\Omega^{\prime}}|u-P|^{1-\gamma} \int_{\Omega^{\prime}}|u-P|^{\gamma}|\nabla u|^{q} d x \\
& \leq M^{\prime} \sup _{\Omega^{\prime}}|u-P|^{1-\gamma} c\left(\int_{\Omega^{\prime}}|\nabla(u-P)|^{p} d x\right)^{n(p-q) /(n-p) p}\left(\int_{\Omega^{\prime}}|\nabla u|^{p} d x\right)^{q / p} \\
& \leq c M^{\prime} \sup _{\Omega^{\prime}}|u-P|^{1-\gamma}\left(\int_{\Omega^{\prime}}|\nabla u|^{p} d x\right)^{(n-q) /(n-p)} \\
& \leq c M^{\prime} D_{p}\left(u, \Omega^{\prime}\right)^{(p-q) /(n-p)} \sup _{\Omega^{\prime}}|u-P|^{1-\gamma} \int_{\Omega^{\prime}}|\nabla u|^{p} d x
\end{aligned}
$$

where we have also used (2.3) and $c$ is a generic constant depending only on $n, p$ and $q$. 
Write $M_{u}=M_{u}\left(\Omega^{\prime}\right)=\sup _{\Omega^{\prime}} u$ and $m_{u}=m_{u}\left(\Omega^{\prime}\right)=\inf _{\Omega^{\prime}} u$. Now $|u-P| \leq$ $M_{u}-m_{u}$ in $\Omega^{\prime}$. Indeed, $\sup _{\Omega^{\prime}} P \leq M_{u}$ and $\inf _{\Omega^{\prime}} P \geq m_{u}$ because if $P(x)>M_{u}$, say, for some $x \in \Omega^{\prime}$, then $u-\min \left(P, M_{u}\right) \in W_{0}^{1, p}\left(\Omega^{\prime}\right)$ and

$$
\int_{\Omega^{\prime}}|\nabla P|^{p} d x>\int_{\Omega^{\prime}}\left|\nabla \min \left(P, M_{u}\right)\right|^{p} d x
$$

which contradicts the minimality of $P$. Hence if $u(x) \geq P(x)$, then

$$
|u(x)-P(x)|=u(x)-P(x) \leq M_{u}-m_{u}
$$

as required and similarly if $u(x)<P(x)$. Since $u$ is continuous, we can for each $x_{o} \in \Omega$ choose $r>0$ so small that

$$
c M\left(M_{u}\left(\Omega^{\prime}\right)-m_{u}\left(\Omega^{\prime}\right)\right)^{1-\gamma} D_{p}\left(u, B\left(x_{o}, r\right)\right)^{(p-q) /(n-p)} \leq \alpha / 2
$$

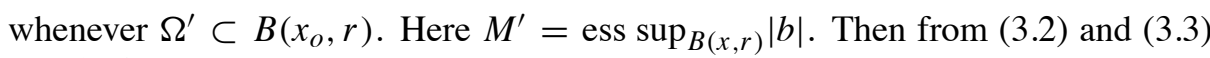
we obtain

$$
\int_{\Omega^{\prime}}|\nabla u|^{p} d x \leq\left(\frac{2 \beta}{\alpha}\right)^{p} \int_{\Omega^{\prime}}|\nabla P|^{p} d x
$$

and this shows that $u \mid B\left(x_{o}, r\right)$ is a $(2 \beta / \alpha)^{p}$-quasiminimizer.

For $p=q, \gamma=0$ and we immediately obtain (3.5) under the assumption

$$
c M\left(M_{u}\left(\Omega^{\prime}\right)-m_{u}\left(\Omega^{\prime}\right)\right) \leq \alpha / 2
$$

and hence $r$ does not depend on $D_{p}\left(u, B\left(x_{o}, r\right)\right)$. This completes the proof.

\section{Examples}

Theorems 2.1 and 2.4 together with [8, Theorem 2.3] and their local versions show that every solution $u$ to equation (1.9) is a local quasiminimizer in $\Omega \subset \mathbf{R}^{n}$, or a quasiminimizer in small sets in the case $u \in W^{1, p}(\Omega)$, whenever

$$
\begin{aligned}
& p>n, p-1 \leq q \leq p, \\
& p=n, n-1 \leq q<n, \\
& p<n, p-1 \leq q \leq p / n+p-1 .
\end{aligned}
$$

Next we consider the remaining ranges of the exponents $p$ and $q$. Let first $0 \leq q<$ $p-1$. Write $s=p-1-q>0$. The function

$$
u_{1}(t)= \begin{cases}-c t^{(p-q) / s}, & t \geq 0, \\ 0, & t<0,\end{cases}
$$

where $c=s^{(p-q) / s}(p-q)^{-1}(p-1)^{-1 / s}>0$ is a distributional solution of the one dimensional Riccati equation $-\left(\left|u^{\prime}\right|^{p-2} u^{\prime}\right)^{\prime}=\left|u^{\prime}\right|^{q}$ in $\mathbf{R}$. The distributional 
property follows from the facts that $u$ is continuously differentiable since ( $p-$ $q) / s>1$ and that $u^{\prime}(0)=0$. The function $u_{1}$ can be extended to $\mathbf{R}^{n}$ as $u(x)=$ $u_{1}\left(x_{1}\right), x=\left(x_{1}, x_{2}, \ldots, x_{n}\right)$, and $u$ is a distributional solution of equation (1.9) in the class $W_{\mathrm{loc}}^{1, p}\left(\mathbf{R}^{n}\right)$. The function $u$ is not a local quasiminimizer because it does not satisfy the strong maximum principle.

For the rest of the cases it is convenient to consider solutions of (1.9) depending only on $|x|$. For a function $u$ depending only on $r=|x|$ the Riccati equation (1.9) in the spherical coordinates of $\mathbf{R}^{n}, n \geq 1$, takes the form

$$
\left|u^{\prime}\right|^{p-2}\left((p-1) u^{\prime \prime}+\frac{n-1}{r} u^{\prime}\right)=-\left|u^{\prime}\right|^{q} .
$$

For $p / n+p-1<q<p$ there are locally unbounded solutions. Indeed, the function $u(x)=c\left(|x|^{(p-q) / s}-1\right)$ is a solution of the class $W_{0}^{1, p}(B(0,1))$ where $s$ is as above and

$$
c=\frac{s(n+s q)^{1 / s}}{q-p}>0 .
$$

Note that $(p-q) / s<0$. The function $u$ cannot be a local quasiminimizer since local quasiminimizers are locally Hölder continuous. Note also that the condition $p / n+p-1<q<p$ implies $p<n$.

Let $q>p$. Now the function

$$
u(x)=c\left(1-|x|^{(p-q) / s}\right)
$$

satisfies equation (1.9) in $B(0,1) \backslash\{0\}$ where $s$ and $c$ are as above. Note that ( $p-$ $q) / s>0$ and $c>0$ whenever

$$
p-1<\frac{n-1}{n} q .
$$

Inequality (4.6) also yields $\nabla u \in L^{q}(B(0,1))$. Moreover $u$ is a distributional solution in $B(0,1)$ and this can be checked writing a function $\varphi \in C_{0}^{\infty}(B(0,1))$ in the form $\varphi=\eta \varphi+(1-\eta) \varphi$ where the function $\eta \in C_{0}^{\infty}(B(0, t))$ satisfies $0 \leq \eta \leq 1$, $\eta=1$ in a neighborhood of 0 and $|\nabla \eta| \leq$ const./ $t$. Then a computation shows that the terms in the formula (1.2) involving the function $\eta \varphi$ approach 0 as $t \rightarrow 0$ because of (4.6). For $p>n$ this fact also follows from [9, Theorem 3.8]. Thus the function $u$ is a bounded $W_{0}^{1, q}(B(0,1))$-solution provided that $q>n$ and (4.6) hold and it has a maximum at 0 . Hence $u$ cannot be a local quasiminimizer in $B(0,1)$.

In the case $p=q<n$ the function

$$
u(x)=(p-1) \log |x|^{(p-n) /(p-1)}
$$

is a locally unbounded $W_{0}^{1, p}(B(0,1))$-solution of equation (1.9) and hence not a local quasiminimizer. The borderline case $p=q=n$ is an interesting case and equation (1.9) is much studied in the plane, see [10] and references therein. The 
two dimensional results are extended to $n \geq 3$ in the recent paper [6]. In particular, the existence result [7, Theorem 2.4] and [6, Theorem 4.3] show that for all $n \geq 2$ equation (1.9) admits locally non-bounded $W_{0}^{1, p}(B(0,1))$-solutions.

Collecting the information from the previous examples and from (4.1)-(4.3) we see that the qualitative quasiminimizing properties of solutions to (1.9) for all the exponents $q \geq 0$ and $p>1$ have been settled except in the wedge domain

$$
\left\{(p, q) \in(1, \infty) \times[0, \infty) \subset \mathbf{R}^{2}: p>n, p<q \leq \frac{n}{n-1}(p-1)\right\} .
$$

\section{References}

[1] F. Della Pietra, "Existence Results for Some Classes of Nonlinear Elliptic Problems", Doctoral thesis, Universita degli Studi di Napoli "Federico II", 2008, http:/www.fedoa.unina.it/2014/1/Della-Pietra-Scienze-Matematiche.pdf

[2] M. Giaquinta, "Introduction to Regularity Theory of Nonlinear Elliptic Systems", Birkhäuser Verlag, Basel, 1993.

[3] M. Giaquinta and E. Giusti, Quasiminima, Ann. Inst. H. Poincaré Anal. Non Linéaire 1 (1984), 79-104.

[4] D. Gilbarg and N. S. Trudinger, "Elliptic Partial Differential Equations of Second Order", second edition, Springer, 1983.

[5] J. Heinonen, T. Kilpeläinen and O. Martio, "Nonlinear Potential Theory of Degenerate Elliptic Equations", Dover, 2006.

[6] T. KilpeläInen, T. KUUSI and A. TUhola-KujanpäÄ, Superharmonic functions are locally renormalized solutions, Ann. Inst. Henri Poincaré, Anal. Non Linéaire 28 (2011), 775-795.

[7] T. KILPELÄINEN and J. MALY, Degenerate elliptic equations with measure data and nonlinear potentials, Ann. Scuola Norm. Sup. Pisa Cl. Sci. (4) 19 (1992), 591-513.

[8] O. MARTIO, Quasilinear Riccati type equations and quasiminimizers, Adv. Nonlinear Stud. 11 (2011), 473-482.

[9] NguYen Cong Phuc, Quasilinear Riccati type equations with super-critical exponents, Comm. Partial Differential Equations 35 (2010), 1958-1981.

[10] A. TUhola-KuJAnPÄ̈̈, A potential theory approach to the equation $-\Delta u=|\nabla u|^{2}$, Ann. Acad. Sci. Fenn. Math. 35 (2010), 633-640.

Department of Mathematics and Statistics FI - 00014 University of Helsinki, Finland olli.martio@acadsci.fi 